\title{
Nutritional Proteomics: Investigating molecular mechanisms underlying the health beneficial effect of functional foods
}

\author{
Yusuke Kawashima ${ }^{1,2}$, Anil Singh ${ }^{1}$, Yoshio Kodera ${ }^{2,3}$, Hiroyuki Matsumoto ${ }^{1}$
}

${ }^{1}$ Department of Biochemistry and Molecular Biology, University of Oklahoma Health Sciences Center, Oklahoma City, OK, USA; ${ }^{2}$ Laboratories of Biomolecular Dynamics, Department of Physics, School of Science, Kitasato University, Kanagawa, Japan; ${ }^{3}$ Center for Disease Proteomics, School of Science, Kitasato University, Kanagawa, Japan

Corresponding author: Hiroyuki Matsumoto, Department of Biochemistry and Molecular Biology, University of Oklahoma Health Sciences Center, Oklahoma City, OK 73104, USA

Submission date: June 23, 2013; Acceptance date: July 19, 2013; Publication date: July 20, 2013

\begin{abstract}
:
Objective: We introduce a new technical and conceptual term "nutritional proteomics" by identifying and quantifying the proteins and their changes in a certain organ or tissue dependent on the food intake by utilizing a mass spectrometry-based proteomics technique.
\end{abstract}

Purpose: Food intake is essentially important for every life on earth to sustain the physical as well as mental functions. The outcome of food intake will be manifested in the health state and its dysfunction. The molecular information about the protein expression change caused by diets will assist us to understand the significance of functional foods. We wish to develop nutritional proteomics to promote a new area in functional food studies for a better understanding of the role of functional foods in health and disease.

Methods: We chose two classes of food ingredients to show the feasibility of nutritional proteomics, omega-3 polyunsaturated fatty acids and omega- 6 polyunsaturated fatty acids both of which are involved in the inflammation/anti-inflammation axis. Each class of the polyunsaturated fatty acids was mixed in mouse chow respectively. The liver tissue of mice fed with omega-3 diet or omega-3 diet was analyzed by the state-of-the-art shotgun proteomics using nano-HPLC-ESI-MS/MS. The data were analyzed by the number of differentially expressed proteins that were guaranteed by $1 \%$ false discovery rate for protein identification and by the statistical significance of variance evaluated by $\mathrm{p}$-value in two-tailed distribution analysis better than $0.05(n=4)$. The differential pattern of protein expression was characterized with Gene Ontology designation.

Results: The data analysis of the shotgun nutritional proteomics identified 2,810 proteins that are validated with $1 \%$ FDR. Among these 2,810 proteins, 125 were characterized with statistical 
significance of variance $(\mathrm{p}<0.05 ; \mathrm{n}=4)$ between the omega- 3 diet and the omega- 6 diet by twotailed distribution analysis. The results illustrate that the dietary influence of omega- 3 and omega- 6 on protein expression is eminent with the proteins directly responsible for catalytic activity in the "Molecular Function" category, totaling 192 proteins, of Gene Ontology designation. In a similar analysis with regard to the "Cellular Localization" category, protein expression changed the most in the sub-categories of "Cytoplasm", "Membrane", "Nucleus", and "Mitochondrion", totaling 221 proteins. The same analysis with regard to "Biological Process" considering the top four categories, i.e., "Metabolic process", "Regulation of biological (process)", "Response to stimulus", and "Transport" also indicated significant alteration of 182 proteins. These results illustrate a robust influence of dietary elements, omega- 3 or omega- 6 polyunsaturated fatty acids, on the protein expression in mouse liver.

Conclusions: Application of nutritional proteomics to the dietary effect of omega-3 polyunsaturated fatty acids compared to that of omega- 6 on mouse liver revealed; 1) significant number of proteins are altered between the two diets dependent on the classes of polyunsaturated fatty acids, omega-3 or omega- 6 , in the diet. The change of protein expression is likely to carry the molecular information that we could possibly decipher, leading to a better understanding of the role of omega-3 polyunsaturated fatty acids in inflammatory/anti-inflammatory process. The results corroborate the concept and utility of nutritional proteomics that should be developed as a part of functional food studies with regard to other dietary types.

Keywords: Nutritional proteomics, functional foods, mass spectrometry, genome database, cellular signaling, omega- 3 and omega- 6 polyunsaturated fatty acids

\section{INTRODUCTION:}

Food intake is essentially important for every life on earth to sustain the physical as well as mental functions. In a classical sense, the functions of foods are to replenish the molecules that construct our body as well as to be consumed as energy source for our everyday activity. From a modern point of view, however, foods can do more than these conventional functions (Martirosyan and Abate, 2010); foods could actively be involved in the healthy maintenance of our body through controlling molecular and cellular signaling pathways. Therefore, investigating the molecular and cellular pathways on which food intake influences will open a fruitful area in functional foods studies. There are at least two ways how to investigate the changes in molecular and cellular pathways caused by diets, one by the genomics method and the other by the proteomics. Genomics measures mRNAs, whereas proteomics measures proteins. These two sets of parameters do not always correlate each other by many unknown reasons (Greenbaum et al., 2003; Gry et al., 2009). Moreover, proteins, but not mRNAs, are the physical entities that function in cellular events. Therefore, it is essentially important to obtain protein information to evaluate the effect of functional foods on the alteration of cellular and molecular signaling. In this report we will introduce a potential subarea in functional food studies; studies of protein expression and its change by diets. We designate the new discipline "nutritional proteomics." In 
this report, we will use a class of essential fatty acids, omega-3 polyunsaturated and omega- 6 PUFAs, as an example of nutritional proteomics study.

All vertebrate animals need to take polyunsaturated fatty acids represented by two major classes, omega-3 and omega-6, from diets. Fish is a good source of omega-3, whereas meats contain high omega-6. Fish, meat, and poultry are the two major groups of protein sources that most of us consume every day unless one is a vegetarian; other sources of PUFAs are some nuts and plant seeds. It is well established that omega-3 acts as anti-inflammatory agent, whereas omega-6 as pro-inflammatory one. It is anticipated, however, that there could be unknown functions of PUFAs other than the regulation of inflammatory state. According to Simopoulos (Simopoulos, 2011a, b). Western diets have drastically changed in the past centuries from being omega-3-dominant to omega-6-dominant. One important factor that caused such dietary change is the modernization of meat industries resulting in the increased consumption of beef, pork, and poultries in everyday life made possible by grain feeding of livestock. Moreover, industrial large-scale production of cooking oils rich in omega- 6 also contributed the dietary changes. Meat and fish diets provide basic building blocks of body such as amino acids and lipids, but, compared to grains, they are not a major source of carbohydrates. Since PUFAs can exist both in their free acid form and in the integrated form in phospholipids such as phosphatidylcholine, phosphatidylserine, and phosphatidylethanolamine and even in other complex lipid types such as cardiolipins, the PUFA molecules are delocalized all over the body, rendering PUFAs a unique characteristic in their influence on health conditions compared to other types of bioactive molecules. It has been well established by the research efforts by many scholars in the past decades that omega-6 and omega-3 PUFAs play important roles in regulating inflammation. Omega-6 represented by arachidonic acid (AA) produce pro-inflammatory agents such as prostagrandins and leukotriens. Cyclo-oxygenases (COXs) play a role in the conversion of AA into pro-inflammatory agents. Most of the non-steroidal anti-inflammatory drugs inhibit the conversion of AA into inflammatory agents by COXs. In contrast, omega-3 produces antiinflammatory agents such as resolvins and protectins (Serhan and Petasis, 2011). In addition it has been recently shown that a class of G-protein-coupled receptor GPR120 binds omega-3 PUFAs and activates the downstream pathway that is responsible for anti-inflammation (Oh et al., 2010); in macrophage, it has been shown that omega-3 suppresses inflammation signals by down-regulating NF- $\kappa \mathrm{B}$. Thus, in addition to the anti-inflammatory action of omega-3 by resolvins and protectins, this type of G-protein-coupled signaling also could participate in the anti-inflammatory pathways activated by the omega-3 class of lipids. The details of these antiinflammatory actions of omega-3 through resolvins, protectins, and GPR120 signaling remain to be elucidated. There have been numerous reports in the past decades describing the health beneficial effects of omega-3 in a wide spectrum of diseases, suggesting that a rich and complex set of cellular signaling pathways underlie the omega-3 effects. Elucidating the role of omega-3 in inflammation will significantly contribute to the understanding of human diseases where inflammation plays an important role in the pathogenesis and in the recovering process of diseases. In this context, nutritional proteomics that investigates protein expression caused by omega-3 and omega-6 PUFAs will certainly promote a better understanding of the complex cellular and molecular events and would give us insights for application of omega-3 to ameliorate disease conditions associated with inflammation. 


\section{MATERIALS AND METHODS:}

C57BL/6 mice: Weight-matched males of C57BL/6 mice at the age of 4 weeks were purchased from The Jackson Laboratory (Bar Harbor, ME). The mice were housed in the OUHSC Rodent Barrier and maintained on a normal mouse chow. After adjustment to the environment for a week, the mice were divided into two groups; HFD6, mouse chow supplemented with $10 \%$ Safflower oil that is rich with omega-6 polyunsaturated fatty acids, and HFD3, mouse chow supplemented with $10 \%$ Menhaden oil that is rich with omega-3 polyunsaturated fatty acids. Both HFD6 and HFD3 diets were adjusted to the energy content to 54\% fat calories with hydrogenated coconut oil in order to accelerate the dietary effects of PUFAs. The rodent barrier had been maintained under temperature- and moisture-controlled pathogen-free environment under a 12 hour light/12 hour dark cycle. After 4 weeks on each diet, we killed the mice ( $n=5$ for each group) by guillotining. The livers were collected, and stored at $-80{ }^{\circ} \mathrm{C}$ until use. The IACUC at OUHSC had approved the animal protocol used in the experiment.

Protein extraction and trypsin digestion: Liver tissues (about $10 \mathrm{mg}$ each dissected from four individual mice; $n=4$ ) were processed individually for shotgun proteomics analysis. The samples were homogenized using an ultrasonic processor in 100 volumes of $12 \mathrm{mM}$ sodium $\mathrm{N}$ lauroylsarcosinate, $12 \mathrm{mM}$ sodium lauryl sulfate, and $200 \mathrm{mM}$ triethyl ammonium bicarbonate containing protease inhibitors (complete mini EDTA-free; Roche Diagnostics GmbH, Mannheim, Germany) according to the published literature (Masuda et al., 2009). The homogenate was centrifuged at $19,000 \mathrm{~g}$ at $4{ }^{\circ} \mathrm{C}$ for $20 \mathrm{~min}$, and the clear supernatant containing proteins was collected. Twenty $\mu \mathrm{L}$ of the supernatant were reduced by the addition of $2 \square \mathrm{L}$ of $200 \mathrm{mM}$ tris(2-carboxyethyl)phosphine for $30 \mathrm{~min}$ at $55^{\circ} \mathrm{C}$. After cooling to room temperature, reduced disulfides were then alkylated by the addition of $2 \mu \mathrm{L}$ of $375 \mathrm{mM}$ iodoacetamide for 30 min at room temperature in the dark. The alkylated sample was enzymatically digested by the addition of $2 \mu \mathrm{L}$ of $100 \mathrm{ng} / \mu \mathrm{L}$ Lys-C (Wako, Osaka, Japan) and $2 \mu \mathrm{L}$ of $100 \mathrm{ng} / \mu \mathrm{L}$ Trypsin (Promega, Madison, WI) for $18 \mathrm{~h}$ at $37^{\circ} \mathrm{C}$. The digested sample was acidified by $5 \%$ trifluoroacetic acid, and the detergents were precipitated. After centrifugation, the supernatant was collected followed by lyophilization. The lyophilized sample was redissolved in $100 \mu \mathrm{L}$ of $1 \times$ Invitrosol (Invitrogen, Carlsbad, CA, USA) in order to enhance the recovery of hydrophobic peptides (Kawashima et al., 2013).

Nano-LC-MS/MS analysis: The peptide digests ( $2 \mu \mathrm{L}$ each) were injected into a trap column: $\mathrm{C}_{18}$ 0.075×50 mm (MonoCap C18 Trap Column; GL Sciences, Tokyo, Japan) followed by an analytical column: $\mathrm{C}_{18} 0.075 \times 120 \mathrm{~mm}$ (Nano HPLC Capillary Column; Nikkyo Technos, Tokyo, Japan), which was interfaced to the EASY-nLC 1000 (Thermo Fisher Scientific, Bremen, Germany). The flow rate of the mobile phase was $300 \mathrm{~nL} / \mathrm{min}$, and the mobile phases consisted of (A) $0.1 \%$ formic acid and (B) $0.1 \%$ formic acid and $90 \%$ acetonitrile. The mobile phase was programmed as follows: 0-8\% B (0-2 min), 8\%-32\% B (2-132 min), 32-45\% B (132-152 min), 45-100\% B (152-153 min), and 100\% B (153-165 min). Separated peptides were introduced from HPLC to Q-Exactive mass spectrometer (Thermo Fisher Scientific). The Q Exactive instrument was operated in the data dependent mode to automatically switch between full scan 
MS and MS/MS acquisition. Full scan MS spectra $(\mathrm{m} / \mathrm{z} 350$ - 1500) were acquired in the Orbitrap with 70,000 resolution at m/z 200 after accumulation of ions to a $3 \times 10^{6}$ target value. The ten most intense peaks with charge state $\geq 2$ from the full scan were selected with an isolation window of 2.0 Da and fragmented in the HCD collision cell with normalized collision energy of $25 \%$. Tandem mass spectra were acquired in the Orbitrap mass analyzer with a mass resolution of 17,500 at $\mathrm{m} / \mathrm{z} 200$ after accumulation of ions to a $2 \times 10^{5}$ target value. The ion selection threshold was $1 \times 10^{5}$ counts, and the maximum allowed ion accumulation times were $60 \mathrm{~ms}$ for full MS scans and $60 \mathrm{~ms}$ for tandem mass spectra. Typical mass spectrometric conditions were as follows: spray voltage, $2 \mathrm{kV}$; no sheath and auxiliary gas flow; heated capillary temperature, $250{ }^{\circ} \mathrm{C}$; dynamic exclusion time, $60 \mathrm{~s}$.

Data analysis: Mass spectral data were processed, exported, and searched against a UniProtKB mouse protein database using MASCOT (version 2.3, Matrixscience, London, UK) by Proteome Discoverer (version 1.3; Thermo Fisher Scientific). Database search parameters were: peptide mass tolerance, $6 \mathrm{ppm}$; fragment tolerance, $0.02 \mathrm{Da}$; enzyme was set to trypsin, allowing up to two missed cleavage; fixed modifications, carbamidomethyl (cysteine); variable modifications, oxidation (methionine). False discovery rate (FDR) (Elias et al., 2005; Weatherly et al., 2005) was calculated by enabling the peptide sequence analysis using a decoy database (http://www.matrixscience.com/help/decoy_help.html). We used 1\% FDR as a cut-off to export results from the analysis. Among over 5,000 proteins identified, 2,810 proteins were validated with better than $1 \%$ FDR. Quantitative analysis of shotgun proteomics data was achieved by label-free quantification (Bantscheff et al., 2007; Lundgren et al., 2010) of four independent liver samples from each group. For this, we used spectral counting (Neilson et al., 2011) after incorporating the mass data to Scaffold (Proteome Software, Inc., Portland, Oregon). The statistical significance of protein expression difference between the two diets (omega-3 and omega- 6 , respectively) was validated by two-tailed distribution analysis $(n=4)$ with the cut off limit of better than $\mathrm{p}<0.05$. The total of 125 proteins passed both the $1 \%$ FDR and the t-test. Assumed molecular functions, cellular localization and biological process of the proteins were defined using Proteome Discoverer according to the Gene Ontology (GO) annotations (http://www.geneontology.org).

\section{RESULTS AND DISCUSSION:}

Novel protocol for nutritional proteomics: The overall protocol of nutritional proteomics using shotgun analysis applied to liver samples is illustrated in Figure 1. In the cohort of C57BL/6 mice we are interested in how protein expression differs between omega-3-enriched diet (HFD3) and omega-6-enriched diet (HFD6) on the high fat (54\% fat calories) background. We put the mice on the high fat background because high fat diets have propensity to induce inflammatory biological response (Todoric et al., 2006), thereby would illustrate better the two opposing effects of omega-3 and omega-6, i.e., the former anti-inflammatory and the latter proinflammatory. Reliability of protein identification was validated with 1\% FDR; after the shotgun data analysis we identified 2,810 proteins that were validated by $1 \%$ FDR for all the liver proteins. Based on the spectral count data, the differential ratio of each protein between HFD6 and HFD3 was calculated. It should be noted that the same protocol could be applied to any 
organ and tissue samples to investigate differences in protein expression caused by different dietary intake.

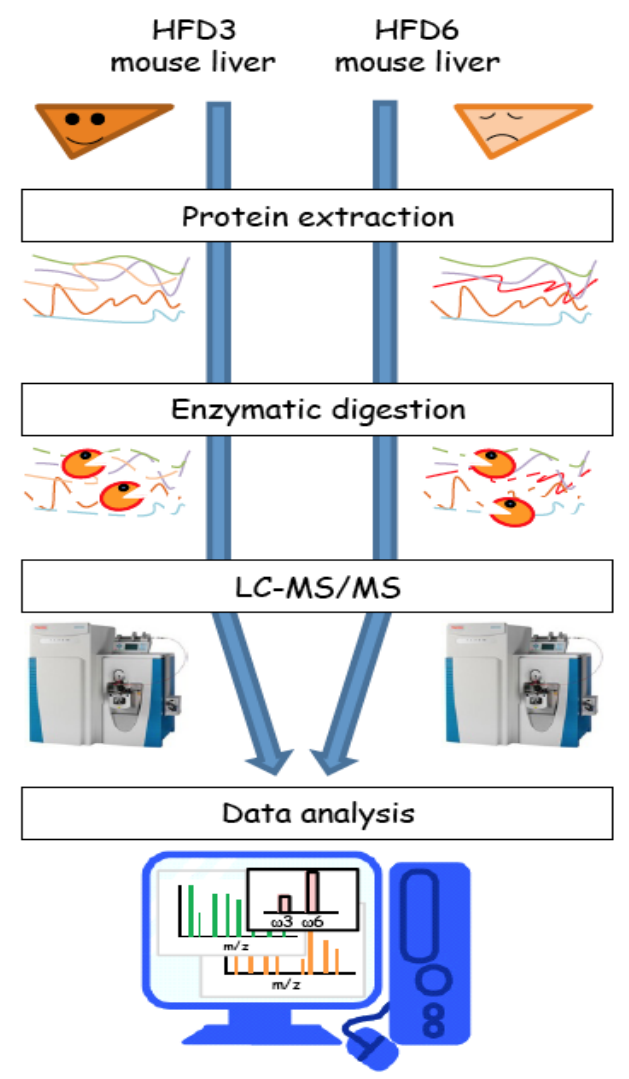

Figure 1. Schematically illustrated protocol for nutritional proteomics. The starting materials are livers from HFD3 (omega-3 diet-fed) or HFD6 (omega-6 diet-fed) C57BL/6 mice. Protein extraction, enzymatic digestion, LC-MS/MS, and data analysis steps are described in Materials and Methods.

Alteration of the cellular signaling pathways caused by dietary intake enriched with omega-3 or omega-6: The data analysis of the shotgun nutritional proteomics outlined in Figure 1 identified 2,810 proteins that are validated with 1\% FDR. Among these 2,810 proteins, 125 were characterized with significant statistical parameters $(\mathrm{p}<0.05 ; \mathrm{n}=4)$. All the 2,810 liver proteins were sorted into three categorical groups, i.e., protein function, cellular localization, and biological process based on the GO annotations (Figure 2). The numbers of proteins that belong to the top four sub-classes are summarized in Table 1. It should be noted that, since many proteins can be counted more than once because one protein could belong to multiple GO classes, the total number of proteins is larger than 2,810 that are validated by FDR scores better than $1 \%$. In the protein function (Figure 2A and Table 1), the proteins in the "Catalytic activity", "Protein binding", "Nucleotide binding", and "Metal ion binding" are counted 3,858. Among these 3,858 proteins, $192(=85+41+40+26)$ changed their expression levels including both up- and downregulation in the top four categories with significant $\mathrm{p}$-values $(\mathrm{p}<0.05)$. These results illustrate that the dietary influence of omega- 3 and omega- 6 on protein expression is eminent with the proteins directly responsible for catalytic activity. We interpret that the follow-ups, i.e., the protein groups classified in "Protein binding", "Nucleotide binding", or "Metal ion binding", are 
entities supporting the functions of the proteins responsible for the catalytic activity. In a similar analysis with regard to cellular localization (Figure 2B and Table 1), protein expression changed the most between the two diets in the GO categories of "Cytoplasm", "Membrane", "Nucleus", and "Mitochondrion", totaling to $221(=101+54+22+44)$ proteins. The same analysis with regard to biological process (Figure 2C and Table 1) considering the top four categories, i.e., "Metabolic process", "Regulation of biological (process)", "Response to stimulus", and "Transport" indicated that $182(=95+31+31+25)$ proteins showed $\mathrm{p}<0.05$. These results clearly illustrate a robust influence of dietary elements, omega- 3 or omega- 6 polyunsaturated fatty acids, on the protein expression. It is anticipated that the substantial alteration of protein expression is a reflection of the complex chains of cellular signaling processes involving the omega-3 and omega- 6 molecules and their in vivo products.

\section{A. Molecular Function}

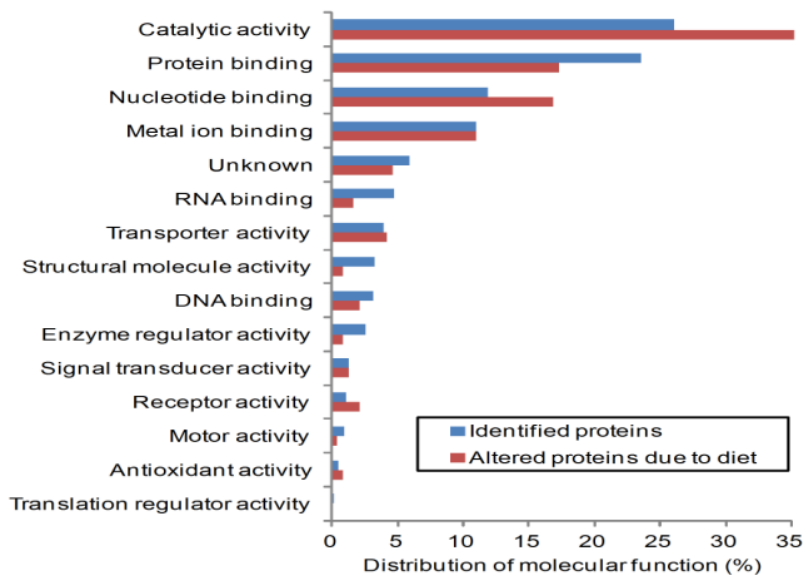

\section{Biological Process}

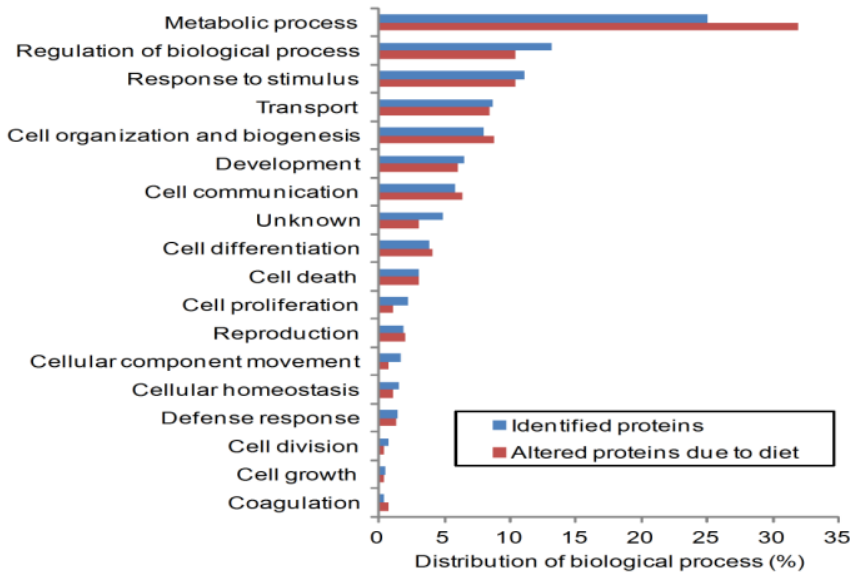

\section{B. Cellular Localization}

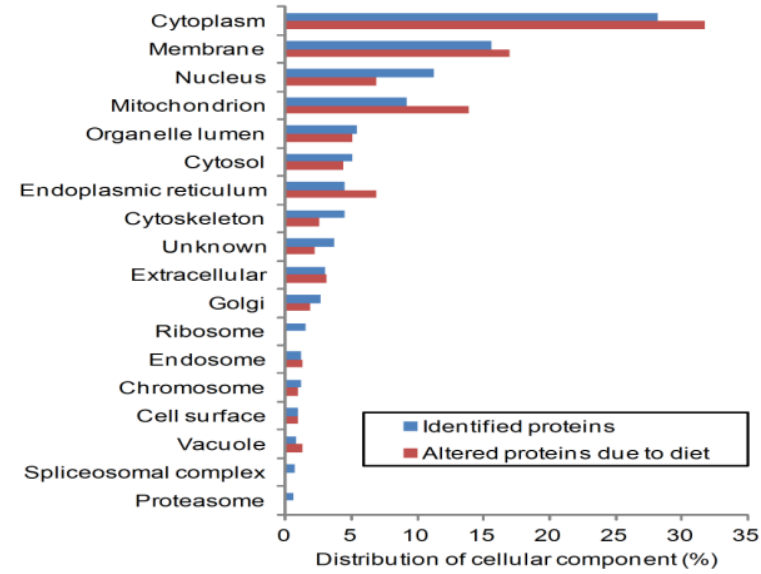

Figure 2. Identified liver proteins and their distribution in categorized groups. In A, B, and C, the identified proteins are shown in blue by the percentage of the total proteins and the proteins that altered expression dependent on diets are shown in red by the percentage of the total changed proteins. Note that the percentage was calculated on different bases between "identified proteins" shown in blue and "altered proteins due to diets" shown in red. A. Categorized by molecular function. B. Categorized by cellular localization. C. Categorized by biological process. 
Almost all available literatures suggest the role of these cellular signaling processes to be associated, directly or indirectly, with inflammation/anti-inflammation events. The result implies that detailed dissection of such complex cascades could be possible using nutritional proteomics approaches.

Table 1. The difference of protein expression between HFD3 and HFD6 is manifested in distinct GO annotation categories.

\begin{tabular}{|c|c|c|c|c|}
\hline Molecular Functiol & $\begin{array}{l}\text { Number of } \\
\text { Identified } \\
\text { Proteins }\end{array}$ & $\begin{array}{c}\text { Number of } \\
\text { Altered } \\
\text { Proteins }\end{array}$ & $\begin{array}{c}\% \text { of } \\
\text { Identified } \\
\text { Proteins }\end{array}$ & $\begin{array}{c}\% \text { of } \\
\text { Altered } \\
\text { Proteins }\end{array}$ \\
\hline Catalytic activity & 1387 & 85 & 26.0 & 35.9 \\
\hline Protein binding & 1255 & 41 & 23.6 & 17.3 \\
\hline Nucleotide binding & 633 & 40 & 11.9 & 16.9 \\
\hline Metal ion binding & 583 & 26 & 10.9 & 11.0 \\
\hline
\end{tabular}

Cellular Localization

\begin{tabular}{|l|c|c|c|c|}
\hline Cytoplasm & 2065 & 101 & 28.1 & 31.8 \\
\hline Membrane & 1142 & 54 & 15.6 & 17.0 \\
\hline Nucleus & 824 & 22 & 11.2 & 6.9 \\
\hline Mitochondrion & 672 & 44 & 9.2 & 13.8 \\
\hline
\end{tabular}

Biological Process

\begin{tabular}{|l|c|c|c|c|}
\hline Metabolic process & 1912 & 95 & 25.1 & 32.0 \\
\hline Regulation of biological & 1006 & 31 & 13.2 & 10.4 \\
\hline Response to stimulus & 843 & 31 & 11.0 & 10.4 \\
\hline Transport & 665 & 25 & 8.7 & 8.4 \\
\hline
\end{tabular}

\section{CONCLUSIONS:}

We showed in this report that the omega-3 diet induced a unique set of liver proteome compared to the one on the omega- 6 diet. Such biochemical phenotypes represented by differential patterns of protein expression are likely to reflect the changes of complex cellular signaling process modulated by the two different types of polyunsaturated fatty acids, omega-3 and omega- 6 . Since all the available information points to the role of omega-3 and omega- 6 in antiinflammatory and inflammatory signaling, the changes in protein expression must reflect the alteration of cellular signaling directly involved in inflammatory/anti-inflammatory processes or of the secondary consequence of such processes. Using nutritional proteomics technique, dissecting the time-dependent sequence of the signaling steps associated with inflammation and modified by diets will be possible. Furthermore, we may be able to extend our observation into more general context; the nutritional proteomics approach will bring in a significant body of information that would help us to decipher the altered cellular signaling process by diets. Also, the nutritional proteomics approach will be applicable to the studies of other functional foods and 
this new discipline could teach us molecular mechanisms how functional foods will affect our health.

Competing interests: Authors have no competing interests with anyone or any organizations.

Author's contributions: Dr. Yusuke Kawashima, Dr. Yoshio Kodera, and Dr. Hiroyuki Matsumoto designed and conducted the study, performed data analysis and interpretation, and drafted, revised and edited the entire manuscript. Anil Singh maintained the mouse colony and prepared liver samples.

Acknowledgments and Funding: This work was supported by OCAST (HR10-120) to H. Matsumoto and by Grant-in-Aid for Scientific Research (B) from the MEXT (No. 22390117) to Y. Kodera. Y. Kawashima was supported by Grant-in-Aid for JSPS Fellows (23-10925).

\section{REFERENCES:}

1. Bantscheff, M., Schirle, M., Sweetman, G., Rick, J., and Kuster, B. (2007). Quantitative mass spectrometry in proteomics: a critical review. Analytical and bioanalytical chemistry 389, 1017-1031.

2. Elias, J.E., Haas, W., Faherty, B.K., and Gygi, S.P. (2005). Comparative evaluation of mass spectrometry platforms used in large-scale proteomics investigations. Nature methods 2 , 667-675.

3. Greenbaum, D., Colangelo, C., Williams, K., and Gerstein, M. (2003). Comparing protein abundance and mRNA expression levels on a genomic scale. Genome biology 4, 117.

4. Gry, M., Rimini, R., Stromberg, S., Asplund, A., Ponten, F., Uhlen, M., and Nilsson, P. (2009). Correlations between RNA and protein expression profiles in 23 human cell lines. BMC genomics 10, 365.

5. Kawashima, Y., Takahashi, N., Satoh, M., Saito, T., Kado, S., Nomura, F., Matsumoto, H., and Kodera, Y. (2013). Enhanced recovery of lyophilized peptides in shotgun proteomics by using an LC-ESI-MS compatible surfactant. Proteomics 13, 751-755.

6. Lundgren, D.H., Hwang, S.I., Wu, L., and Han, D.K. (2010). Role of spectral counting in quantitative proteomics. Expert review of proteomics 7, 39-53.

7. Martirosyan, D.M., and Abate, N. (2010). Functional Foods for Chronic Diseases, Volume 5: Diabetes and Diabetes related Diseases, Vol 5 (Richardson, TX: Food Science Publisher).

8. Masuda, T., Saito, N., Tomita, M., and Ishihama, Y. (2009). Unbiased quantitation of Escherichia coli membrane proteome using phase transfer surfactants. Molecular \& cellular proteomics : MCP 8, 2770-2777.

9. Neilson, K.A., Ali, N.A., Muralidharan, S., Mirzaei, M., Mariani, M., Assadourian, G., Lee, A., van Sluyter, S.C., and Haynes, P.A. (2011). Less label, more free: approaches in labelfree quantitative mass spectrometry. Proteomics 11, 535-553.

10. Oh, D.Y., Talukdar, S., Bae, E.J., Imamura, T., Morinaga, H., Fan, W., Li, P., Lu, W.J., Watkins, S.M., and Olefsky, J.M. (2010). GPR120 is an omega-3 fatty acid receptor mediating potent anti-inflammatory and insulin-sensitizing effects. Cell 142, 687-698. 
11. Serhan, C.N., and Petasis, N.A. (2011). Resolvins and protectins in inflammation resolution. Chemical reviews 111, 5922-5943.

12. Simopoulos, A.P. (2011a). Evolutionary aspects of diet: the omega-6/omega-3 ratio and the brain. Molecular neurobiology 44, 203-215.

13. Simopoulos, A.P. (2011b). Importance of the omega-6/omega-3 balance in health and disease: evolutionary aspects of diet. World review of nutrition and dietetics 102, 10-21.

14. Todoric, J., Loffler, M., Huber, J., Bilban, M., Reimers, M., Kadl, A., Zeyda, M., Waldhausl, W., and Stulnig, T.M. (2006). Adipose tissue inflammation induced by high-fat diet in obese diabetic mice is prevented by n-3 polyunsaturated fatty acids. Diabetologia 49, 2109-2119.

15. Weatherly, D.B., Atwood, J.A., 3rd, Minning, T.A., Cavola, C., Tarleton, R.L., and Orlando, R. (2005). A Heuristic method for assigning a false-discovery rate for protein identifications from Mascot database search results. Molecular \& cellular proteomics : MCP $4,762-772$. 